\title{
A clinical model to predict the risk of liver metastases in newly diagnosed ovarian cancer: a population-based study
}

\author{
Yufei Yuan ${ }^{1}$, Ruoran Wang ${ }^{2}$, Fanfan Guo ${ }^{1}$, Yidan Zhang ${ }^{1}$, Hongyan Wang ${ }^{1}$, Xia Li $^{1}$, Gui-Qin Bai ${ }^{3}$ \\ ${ }^{1}$ Medicine Department, Xi'an Jiaotong University, Xi'an, China; ${ }^{2}$ Department of Critical Care Medicine, West China Hospital of Sichuan University, \\ Chengdu, China; ${ }^{3}$ Department of Obstetrics and Gynecology, The First Affiliated Hospital of Xi'an Jiaotong University, Xi'an, China \\ Contributions: (I) Conception and design: Y Yuan; (II) Administrative support: G Bai; (III) Provision of study materials or patients: F Guo; (IV) \\ Collection and assembly of data: Y Yuan, Y Zhang; (V) Data analysis and interpretation: R Wang; (VI) Manuscript writing: All authors; (VII) Final \\ approval of manuscript: All authors. \\ Correspondence to: Gui-Qin Bai. The First Affiliated Hospital of Xi’an Jiaotong University, Yanta West Road, Xi’an, Shaanxi, China. Email: baigq@126.com.
}

Background: Liver metastases are important in determining the prognosis of ovarian cancer. We aimed to develop and validate nomograms to predict the risk of liver metastases in patients with early-stage ovarian cancer.

Methods: A total of 13,487 patients were enrolled in the study based on their records in the Surveillance, Epidemiology, and End Results (SEER) database. Risk factors of liver metastases were assessed based on univariable and multivariable logistic regression. A nomogram was also formulated based on the results of multivariable logistic analysis. The area under the receiver-operating characteristic curve was calculated to evaluate the discrimination abilities of the metastasis-related factors and liver metastases nomogram. A calibration plot was generated to analyze the consistency between the observed probability and predicted probability of liver metastases in patients with ovarian cancer.

Results: Four related factors were determined based on univariable and multivariable logistic regression, including the T1 stage, N1 stage, and presence of lung and bone metastases. The liver metastases nomogram composed of four features could be used to determine the prediction effect. The calibration plot showed good consistency between the nomogram prediction and actual observation. The receiver-operating characteristic curve showed that the forecast nomogram exhibited a good forecast value.

Conclusions: This clinical prediction model has high accuracy to identify patients with newly diagnosed ovarian cancer who carry a high risk of liver metastases and provide a personalized treatment plan for these patients.

Keywords: Ovarian cancer; liver metastases; nomogram; receiver-operating characteristic curve; Surveillance, Epidemiology, End Results (SEER) database

Submitted Jun 14, 2020. Accepted for publication Sep 20, 2020.

doi: $10.21037 /$ tcr-20-2321

View this article at: http://dx.doi.org/10.21037/tcr-20-2321

\section{Introduction}

Ovarian cancer is one of the most common malignant tumors that affects the female reproductive system. It is estimated that 21,750 women in the US will be diagnosed with ovarian cancer. Ovarian cancer is the fifth most common cause of cancer-related deaths among American women. It is estimated that 13,940 individuals will die from this disease this year (1). Because the symptoms are unclear and there is currently no effective screening method, most

\footnotetext{
$\wedge$ ORCID: 0000-0001-7229-9361.
} 
patients are already harboring the advanced stage (III and IV) when they are diagnosed (2).

Ovarian cancer is called the "silent killer". It is estimated that only $15 \%$ of ovarian cancers are restricted to the ovaries, whereas $17 \%$ are localized metastases, and $62 \%$ are associated with distant metastases. Ovarian cancer can spread through the intraperitoneal, lymphatic, and bloodborne pathways (3). The most common distant metastatic site is the liver, followed by the distant lymph nodes, lungs, bones, and brain (4). Distant metastases to the liver, lungs, brain, and bones are associated with poor overall survival $(5,6)$. The median survival time from the diagnosis of distant metastases is only 4 months $(7,8)$. Therefore, early detection of liver metastases from ovarian cancer is important for modifying treatment strategies and improving patient prognosis.

Most studies of ovarian cancer metastases use liver metastases to predict the prognosis and recurrence of ovarian cancer (9-11). Little is known about the clinical and pathological risk factors of liver metastases in patients with early-stage ovarian cancer. Therefore, we aimed to predict the risk factors based on statistical prediction models.

The National Cancer Institute's Surveillance, Epidemiology, and End Results (SEER) database is the largest publicly available cancer dataset, covering approximately $30 \%$ of the US population. It regularly records data on patient demographic information, tumor characteristics, general treatment, and survival time, and important information status updates are provided every year. We aimed to use nomograms to evaluate patients with early-stage ovarian cancer, identify patients with high risk scores, and help modify treatment strategies in clinical applications.

We present the following article in accordance with the TRIPOD reporting checklist (available at http://dx.doi. org/10.21037/tcr-20-2321).

\section{Methods}

\section{Data source}

The SEER database includes information on demographics, cancer incidence, and survival outcomes from populationbased registries for approximately $30 \%$ of the US population. Data of this study were obtained from the SEER program of the National Cancer Institute using the Surveillance, Epidemiology, and End Results *Stat software (version 8.3.5). The study was conducted in accordance with the Declaration of Helsinki (as revised in 2013). The research was exempt from ethics statement as the SEER is a publicly available database, and data extracted from SEER were identified as an exempted study. Since the data collected from the SEER database were anonymized and de-identified prior to release, informed patient consent was not required in our study.

\section{Study population}

Data were obtained from the National Cancer Institute's SEER program between 2010 and 2014, as the statuses of liver metastases and other sites of distant metastases were collected in the SEER database from 2010, and the data were last updated on December 31, 2014. Initially, 29,313 patients diagnosed with ovarian cancer were identified in the database. After excluding 15,826 unqualified cases, we finally collected 13,487 patients with ovarian cancer. The flowchart of the subjects' selection is illustrated in Figure 1. We collected patient demographics and tumor variables. The demographic variables included age, race, marital status, insurance status, and household income at the time of diagnosis. The tumor variables included laterality; tumor grade; tumor size; histological type; whether the disease was stage $\mathrm{T}$ or $\mathrm{N}$; and whether bone, brain, liver, and lung distant metastases occurred at the time of initial diagnosis.

\section{Statistical analysis}

Statistical analysis was performed using SPSS 21 software. Categorical data were presented as frequencies (\%) and analyzed using the chi-squared test. The KolmogorovSmirnov test was used to verify the normality of the variables. Normally distributed variables were expressed as means \pm standard deviations, while non-normally distributed variables were expressed as medians (interquartile ranges). Univariable and multivariable logistic regression analyses were used to determine the risk factors of liver metastases in patients with ovarian cancer. Factors with a $\mathrm{P}$ value less than 0.05 were incorporated into the multivariable regression model.

A nomogram was also formulated based on the results of multivariable logistic analysis using the rms package in $\mathrm{R}$ version 3.4.1 (R Foundation for Statistical Computing, Vienna, Austria; www.r-project.org). The receiver-operating characteristic (ROC) curve was generated, and the area under the ROC curve (AUC) was calculated to evaluate the discrimination abilities of the metastasis-related factors and liver metastases nomogram. Finally, we evaluated the 


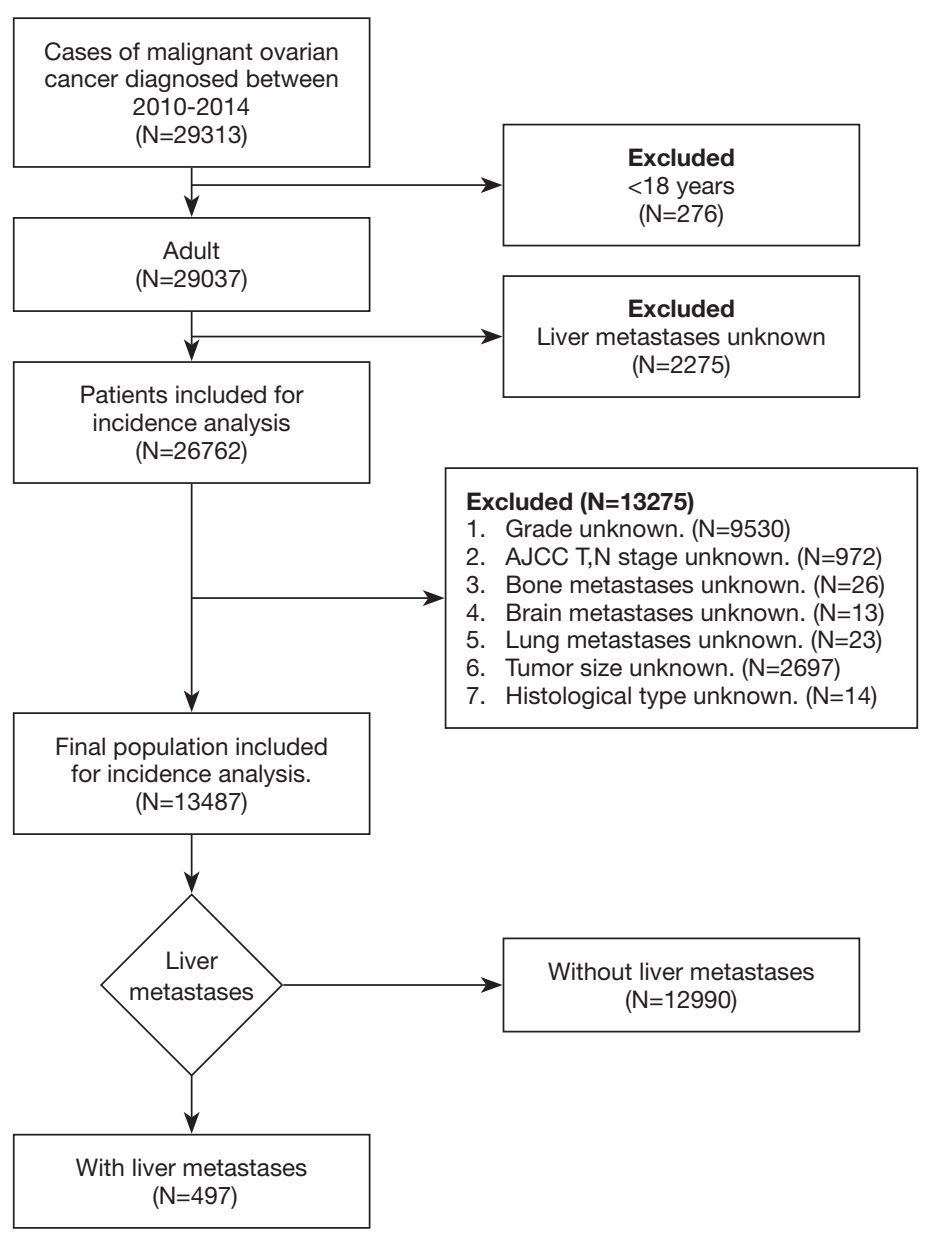

Figure 1 Study flowchart.

stability of the prognostic nomogram by internal validation using 1,000 bootstrap samples. A calibration plot was generated to analyze the consistency between the observed probability and predicted probability of liver metastases in patients with ovarian cancer.

\section{Results}

\section{Demographic and clinical characteristics}

A total of 13,487 patients with ovarian cancer met the inclusion criteria. The median age of the patients was 59 years (51-68 years). Among these patients, 487 (3.61\%) patients with liver metastases had a median age of 63 years (54-71 years). The demographic and clinical characteristics of the included patients are shown in Table 1. Age $(\mathrm{P}<0.001)$, tumor grade $(\mathrm{P}<0.001)$, laterality $(\mathrm{P}<0.001)$, American Joint Committee on Cancer $\mathrm{T}$ stage $(\mathrm{P}<0.001)$ and $\mathrm{N}$ stage
$(\mathrm{P}<0.001)$, histological type $(\mathrm{P}<0.001)$, bone metastases $(\mathrm{P}<0.001)$, brain metastases $(\mathrm{P}=0.017)$, and lung metastases $(\mathrm{P}<0.001)$ exhibited significant differences. There were no statistically significant differences in race $(\mathrm{P}=0.232)$, year of diagnosis $(\mathrm{P}=0.462)$, household income $(\mathrm{P}=0.103)$, marital status $(\mathrm{P}=0.274)$, insurance status $(\mathrm{P}=0.308)$, and tumor size $(\mathrm{P}=0.463)$.

\section{Risk factors of developing liver metastases}

Univariable logistic analysis showed that advanced age, bilateral tumors, N1 stage, poorly differentiated and undifferentiated grade, lung metastases, bone metastases, and brain metastases were all positively associated with a risk of developing liver metastases. Non-serous histology and T1 stage were negatively related to liver metastases (Table 2).

Multivariable logistic regression indicated that the T1 
Table 1 Demographical and clinical characteristics between patient with liver metastases and patients without liver metastases

\begin{tabular}{|c|c|c|c|c|}
\hline Variables & All patients $(\mathrm{N}=13,487)$ & $\begin{array}{l}\text { Patients with liver } \\
\text { metastases }(\mathrm{N}=487)\end{array}$ & $\begin{array}{l}\text { Patients without liver } \\
\text { metastases }(\mathrm{N}=12,990)\end{array}$ & $P$ value \\
\hline Age & $59(51-68)$ & $63(54-71)$ & $59(50-68)$ & $<0.001$ \\
\hline White & $11,142(82.6 \%)$ & $416(83.7 \%)$ & $10,726(82.6 \%)$ & \\
\hline Unknown & $54(0.4 \%)$ & $1(0.2 \%)$ & $53(0.4 \%)$ & \\
\hline Marital status & & & & 0.274 \\
\hline Separated & $2,964(22.0 \%)$ & $126(25.4 \%)$ & $2,838(21.8 \%)$ & \\
\hline Unknown & $548(4.1 \%)$ & $18(3.6 \%)$ & $530(4.1 \%)$ & \\
\hline Insurance status & & & & 0.308 \\
\hline Uninsured & $501(3.7 \%)$ & $13(2.6 \%)$ & $488(3.8 \%)$ & \\
\hline Insured & $12,845(95.2 \%)$ & 477 (96.0\%) & $12,368(95.2 \%)$ & \\
\hline Unknown & $141(1.0 \%)$ & $7(1.4 \%)$ & $134(1.0 \%)$ & \\
\hline Household income & $70,296(63,636-87,648)$ & $67968(60,816-82,848)$ & $70,296(63,900-87,648)$ & 0.103 \\
\hline Year of diagnosis & & & & 0.462 \\
\hline Tumor size & & & & 0.463 \\
\hline$<2 \mathrm{~cm}$ & $1,116(8.3 \%)$ & $36(7.2 \%)$ & $1,080(8.3 \%)$ & \\
\hline $2-5 \mathrm{~cm}$ & $2,246(16.7 \%)$ & $91(18.3 \%)$ & $2,155(16.6 \%)$ & \\
\hline$>5 \mathrm{~cm}$ & $10,125(75.1 \%)$ & $370(74.4 \%)$ & $9,755(75.1 \%)$ & \\
\hline Laterality & & & & $<0.001$ \\
\hline Left & $4,086(30.3 \%)$ & $105(21.1 \%)$ & 3,981 (30.6\%) & \\
\hline Right & $4,132(30.6 \%)$ & 97 (19.5\%) & $4,035(31.1 \%)$ & \\
\hline Bilateral & $5,044(37.4 \%)$ & $268(53.9 \%)$ & $4,776(36.8 \%)$ & \\
\hline Others/unknown & $225(1.7 \%)$ & 27 (5.4\%) & $198(1.5 \%)$ & \\
\hline Grade & & & & $<0.001$ \\
\hline Well differentiated & 1,662 (12.3\%) & $13(2.6 \%)$ & $1,649(12.7 \%)$ & \\
\hline
\end{tabular}

Table 1 (continued) 
Table 1 (continued)

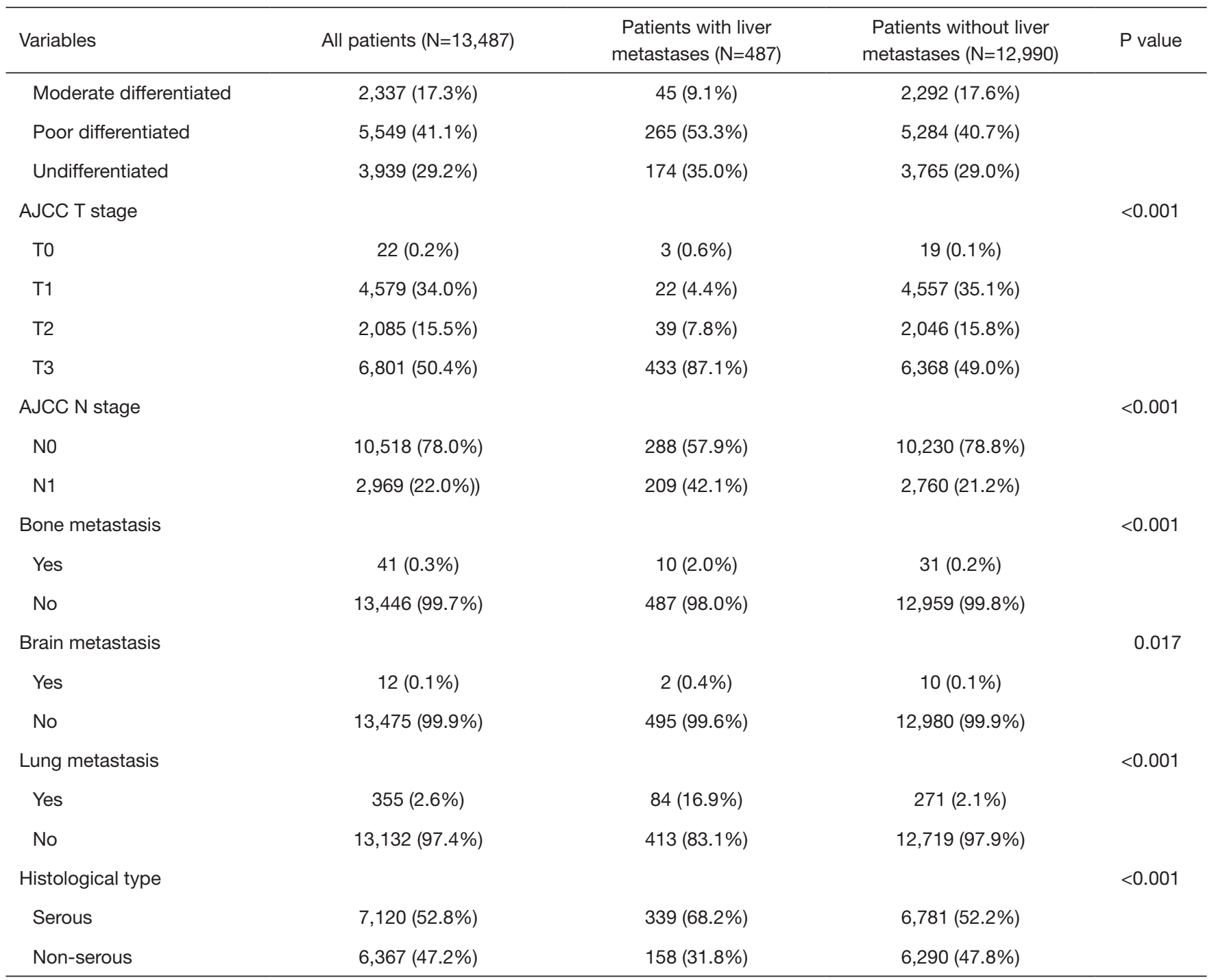

stage was negatively associated with liver metastases, while the N1 stage and presence of lung and bone metastases were positively associated with liver metastases development. Statistically significant factors in multivariable logistic regression were used to develop risk models for predicting liver metastases (Table 2).

\section{Liver metastases nomogram for ovarian cancer}

Through the logistic regression model, we built a liver metastasis nomogram incorporating the aforementioned independent metastases factors for visualization and facilitating clinical practice, as shown in Figure $2 A$. We used bootstrapping to internally validate the model. Stability and internal validation were studied using 1,000 bootstrap samples. The calibration plot, displayed in Figure 2B, for the probability of liver metastases after diagnosis, showed good consistency between the nomogram predictions and actual observations.

\section{ROC curve analysis and predictive value assessment}

The ROC curve was plotted to determine the predictive value of the nomogram for the presence of liver metastases in patients with ovarian cancer. As shown in Figure 3, the AUCs of the presence of lung and bone metastases, T1 stage, and N1 stage were 0.765 , respectively, indicating their good predictive value. 
Table 2 Univariable and Multivariable Logistic Regression for analyzing the associated factors for developing liver metastases in ovarian cancer patients

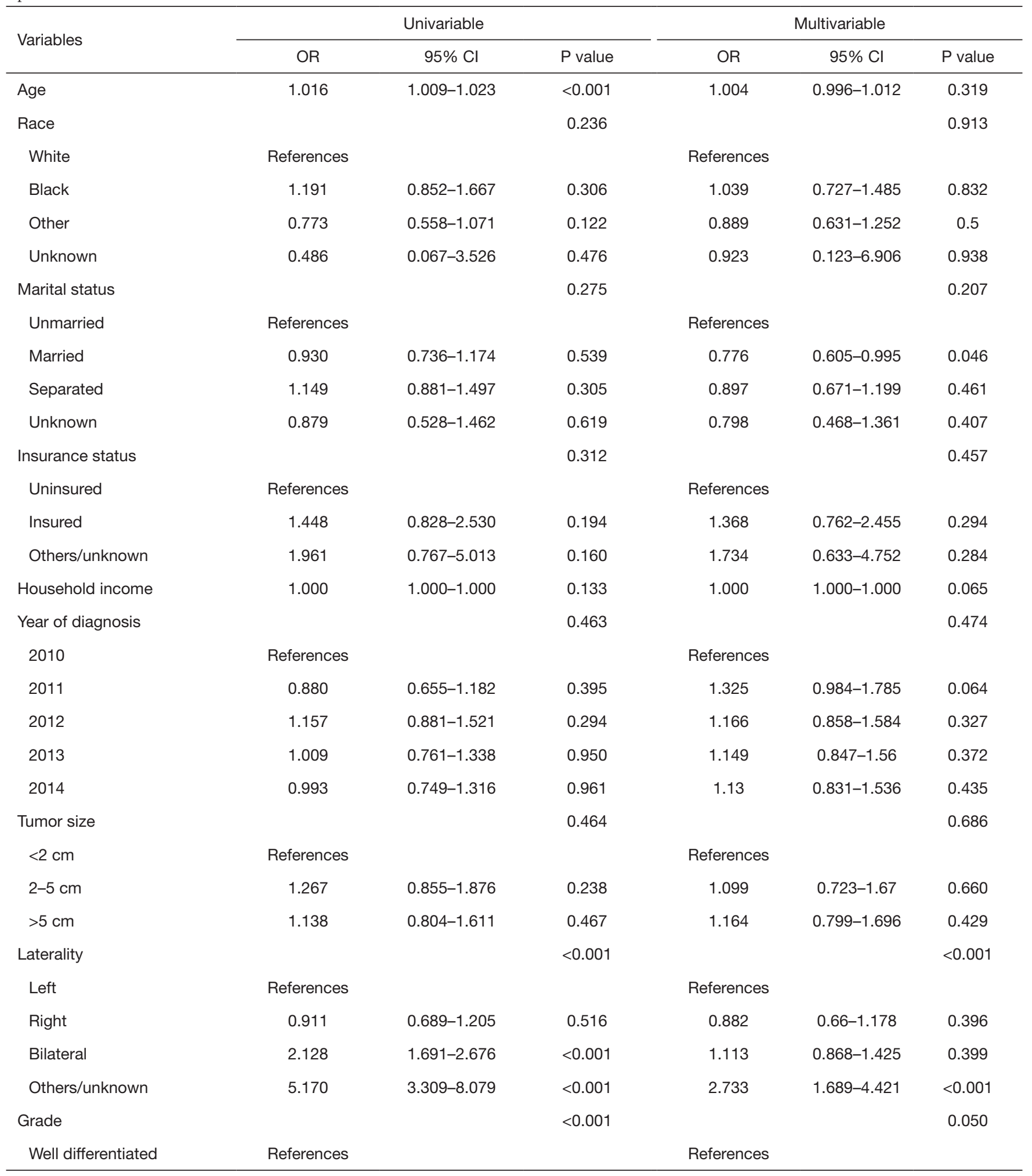

Table 2 (continued) 
Table 2 (continued)

\begin{tabular}{|c|c|c|c|c|c|c|}
\hline Variables & \multicolumn{3}{|c|}{ Univariable } & \multicolumn{3}{|c|}{ Multivariable } \\
\hline Moderate differentiated & 2.490 & $1.339-4.631$ & 0.004 & 1.432 & $0.758-2.702$ & 0.268 \\
\hline Poor differentiated & 6.362 & $3.636-11.131$ & $<0.001$ & 1.931 & $1.077-3.463$ & 0.027 \\
\hline Undifferentiated & 5.862 & $3.327-10.330$ & $<0.001$ & 1.661 & $0.918-3.006$ & 0.094 \\
\hline TO & References & & & References & & \\
\hline $\mathrm{T} 1$ & 0.031 & $0.008-0.111$ & $<0.001$ & 0.152 & $0.036-0.639$ & 0.01 \\
\hline T2 & 0.121 & $0.034-0.425$ & 0.001 & 0.447 & $0.111-1.81$ & 0.259 \\
\hline T3 & 0.431 & $0.127-1.461$ & 0.176 & 1.4 & $0.358-5.48$ & 0.629 \\
\hline N1 & 2.690 & $2.240-3.230$ & $<0.001$ & 1.490 & $1.228-1.808$ & $<0.001$ \\
\hline Bone metastasis & & & $<0.001$ & & & 0.001 \\
\hline No & References & & & References & & \\
\hline Yes & 8.584 & $4.184-17.609$ & $<0.001$ & 4.287 & $1.878-9.787$ & 0.001 \\
\hline Brain metastasis & & & 0.33 & & & 0.306 \\
\hline No & References & & & References & & \\
\hline Yes & 5.244 & 1.146-23.999 & 0.33 & 2.51 & $0.463-13.597$ & 0.286 \\
\hline Lung metastasis & & & $<0.001$ & $<0.001$ & & $<0.001$ \\
\hline
\end{tabular}

\section{Discussion}

Liver metastases are common in patients with ovarian cancer. Previous studies have shown that liver metastases are found in up to $50 \%$ of patients who die from ovarian cancer (12). Liver metastases are the main cause of death from ovarian cancer. Chemotherapy is currently the main treatment for ovarian cancer with metastases. However, studies have shown that performing cytoreductive surgery, including hysterectomy, bilateral salpingo-oophorectomy, omentum resection, and resection of all metastatic lesions, is beneficial to the survival of patients (13). Performing complete resection of liver metastases can confer benefits to survival (14). A patient's disease-free survival and overall survival improve (15). Valerio Gallotta's study found that hepatic resection during the second cytoreductive surgery was beneficial for patients with recurrent ovarian cancer, and BRCA gene mutations were associated with better progression-free survival after hepatic resection (16). It was feasible and safe to apply laparoscopy to remove the lesions in the abdominal cavity including the liver, allowing patients to benefit (17). Therefore, early detection of liver metastasis, clarification of the molecular characteristics of tumors, and selection of appropriate multi-channel treatment are of great significance to improve the prognosis of patients with ovarian cancer. 


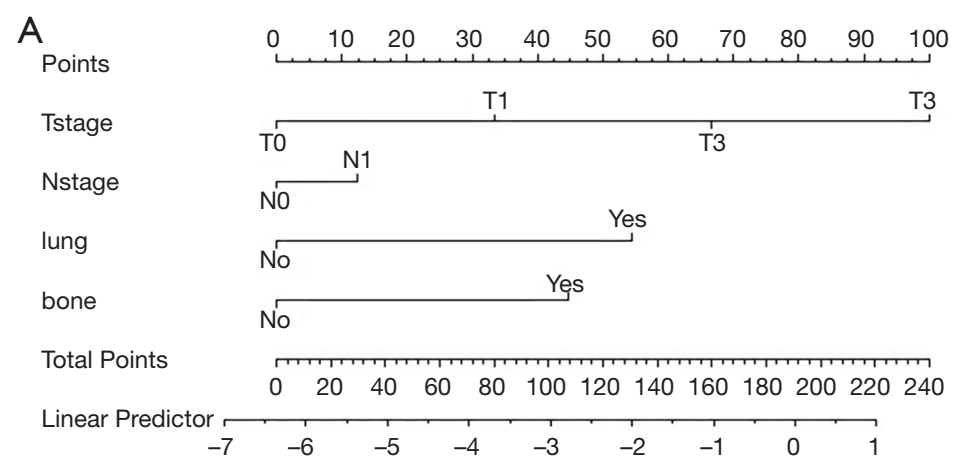

B

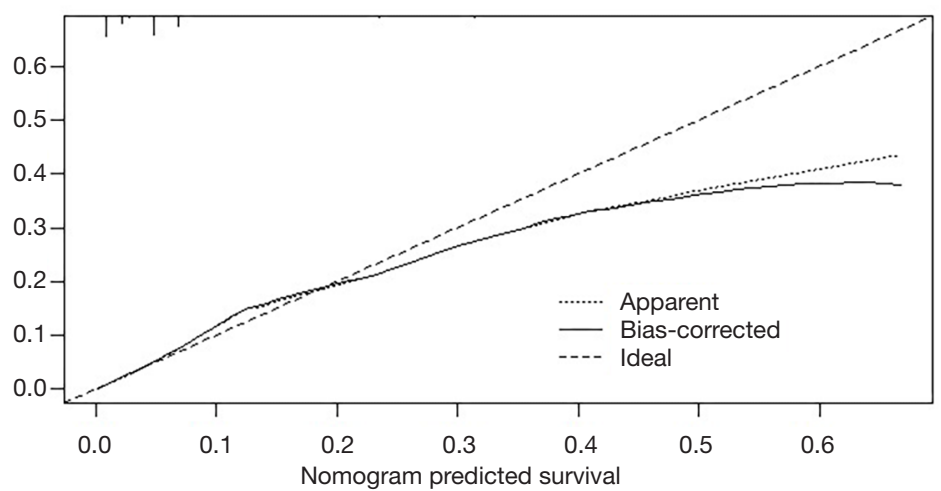

Figure 2 The nomogram and the calibration curve for liver metastasis in patients with ovarian cancer. (A) The nomogram for liver metastasis in patients with ovarian cancer. The total point score is projected on the bottom scales to determine the probability of cancer metastasis in an individual; (B) the calibration curves for predicting liver metastases.

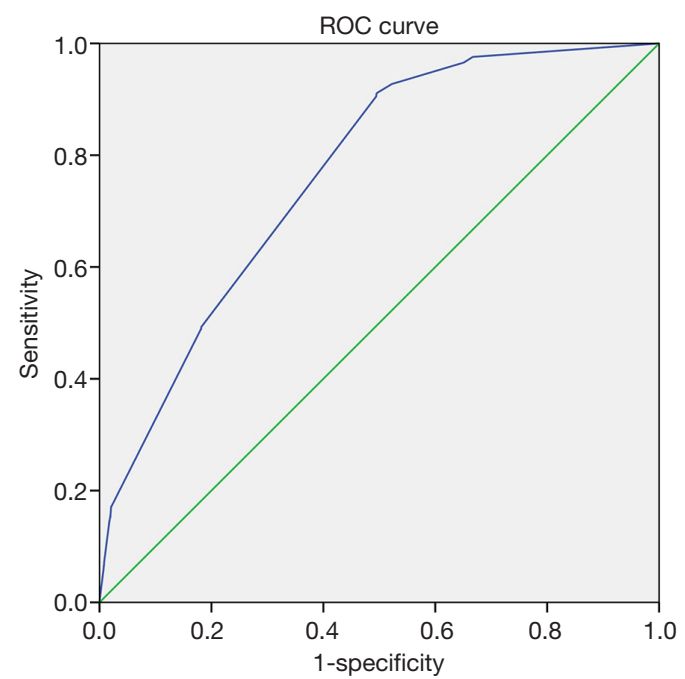

Figure 3 The ROC curve. The sensitivity of the ROC curve is 0.911 and the specificity is 0.504 .
In order to better solve this problem, this study is the first to generate a risk model based on clinical and tumor characteristics through the population-based SEER database to predict the risk of liver metastases in newly diagnosed patients with ovarian cancer. We found a series of risk factors associated with the development of liver metastases in patients with ovarian cancer, including a high $\mathrm{T}$ stage (T1) and $\mathrm{N}$ stage (N1) and the presence of lung and bone metastases. Deng et al.'s study showed that poor differentiation and lymph node involvement are positively correlated with the occurrence of distant metastases (5). Our study findings were in line with these findings. In the univariable logistic regression, we found that the degree of differentiation and N1 stage were positively correlated with liver metastases. In the multivariable logistic regression, the correlation between differentiation and liver metastases was not statistically significant. Previous studies have shown 
that the histological type of ovarian cancer is related to the occurrence of bone metastases in patients with ovarian cancer and their prognosis $(9,18)$. In the univariable logistic regression, we found that serous ovarian cancer was more prone to liver metastases than non-serous ovarian cancer. Bone metastases and brain metastases are independent risk factors affecting the prognosis of patients with ovarian cancer (6). Our study found that bone metastases and lung metastases were risk factors of liver metastases. If a patient with ovarian cancer harbors bone metastases and lung metastases, the cancer cells are more prone to spread. Multivariable logistic regression has shown that the T1 stage is negatively correlated with liver metastases. The T1 stage is associated with the tumor being limited to the ovaries, with no ovarian surface, pelvic, extraperitoneal, or peritoneal metastasis. Therefore, the risk of liver metastasis is low (19). Zecchin et al. found that tumor size affects the prognosis of patients with ovarian cancer (20). Our study did not reveal a relationship between tumor size and liver metastases.

We constructed a nomogram for ovarian cancer liver metastases and verified the results. It can be used to predict the risk of liver metastases in patients with ovarian cancer. The nomogram of liver metastases included 4 factors: $\mathrm{T}$ stage, $\mathrm{N}$ stage, whether bone metastases occur, and whether lung metastases occur. The nomogram showed good consistency between the predicted and observed results in the verification. In addition, the area under the ROC curve was 0.765 , which also showed good diagnostic efficiency. For patients whose risk of metastases is predicted to be higher by this model, periodic computed tomography scans can be considered for prevention and care, so as to better guide clinical procedures.

This study has several limitations. First, in this study, the existence or absence of liver metastases was analyzed based only on preliminary diagnosis. Because the liver metastases that appear later in the disease course may not be recorded in the SEER database, patients with late-stage liver metastases cannot be analyzed. Second, this model does not include some clinical indicators and genetic markers, and biomarkers can be included to improve the accuracy of the model. Third, because the patients in this study were from the American population, further verification is needed in populations of other countries to apply the risk prediction model more accurately.

Liver metastases are the most common distant metastatic site of ovarian cancer. Early detection of liver metastases through routine screening at the initial diagnosis of ovarian cancer will be beneficial for patients carrying a high risk. Here, this clinical prediction model has high accuracy to identify patients with newly diagnosed ovarian cancer who carry a high risk of liver metastases and provide a personalized treatment plan for these patients.

\section{Acknowledgments}

The authors acknowledge the great efforts of the Surveillance, Epidemiology, and End Results Program in the creation of the Surveillance, Epidemiology, and End Results database. We would like to thank Editage (www. editage.cn) for English language editing.

Funding: None.

\section{Footnote}

Reporting Checklist: The authors have completed the TRIPOD reporting checklist. Available at http://dx.doi. org/10.21037/tcr-20-2321

Conflicts of Interest: All authors have completed the ICMJE uniform disclosure form (available at http://dx.doi. org/10.21037/tcr-20-2321). The authors have no conflicts of interest to declare.

Ethical Statement: The authors are accountable for all aspects of the work in ensuring that questions related to the accuracy or integrity of any part of the work are appropriately investigated and resolved. The study was conducted in accordance with the Declaration of Helsinki (as revised in 2013). The research was exempt from ethics statement as the SEER is a publicly available database, and data extracted from SEER were identified as an exempted study. Since the data collected from the Surveillance, Epidemiology, and End Results database were anonymized and de-identified prior to release, informed patient consent was not required in our study.

Open Access Statement: This is an Open Access article distributed in accordance with the Creative Commons Attribution-NonCommercial-NoDerivs 4.0 International License (CC BY-NC-ND 4.0), which permits the noncommercial replication and distribution of the article with the strict proviso that no changes or edits are made and the original work is properly cited (including links to both the formal publication through the relevant DOI and the license). See: https://creativecommons.org/licenses/by-nc-nd/4.0/. 


\section{References}

1. DeSantis CE, Ma J, Gaudet MM, et al. Breast cancer statistics, 2019. CA Cancer J Clin 2019;69:438-51.

2. Siegel RL, Miller KD, Jemal A. Cancer statistics, 2019. CA Cancer J Clin 2019;69:7-34.

3. Jelovac D, Armstrong DK. Recent progress in the diagnosis and treatment of ovarian cancer. CA Cancer J Clin 2011;61:183-203.

4. Bergman F. Carcinoma of the ovary. A clinicopathological study of 86 autopsied cases with special reference to mode of spread. Acta Obstet Gynecol Scand 1966;45:211-31.

5. Deng K, Yang C, Tan Q, et al. Sites of distant metastases and overall survival in ovarian cancer: A study of 1481 patients. Gynecol Oncol 2018;150:460-5.

6. Gardner AB, Charo LM, Mann AK, et al. Ovarian, uterine, and cervical cancer patients with distant metastases at diagnosis: most common locations and outcomes. Clin Exp Metastasis 2020;37:107-13.

7. Dauplat J, Hacker NF, Nieberg RK, et al. Distant metastases in epithelial ovarian carcinoma. Cancer 1987;60:1561-6.

8. O'Neill AC, Somarouthu B, Tirumani SH, et al. Patterns and Prognostic Importance of Hepatic Involvement in Patients with Serous Ovarian Cancer: A Single-Institution Experience with 244 Patients. Radiology 2017;282:160-70.

9. Wu SG, Li FY, Lei J, et al. Histological Tumor Type is Associated with One-Year Cause-Specific Survival in Women with Stage III-IV Epithelial Ovarian Cancer: A Surveillance, Epidemiology, and End Results (SEER) Database Population Study, 2004-2014. Med Sci Monit 2020;26:e920531.

10. Luo P, Zhou JG, Jin SH, et al. Influence of marital status on overall survival in patients with ovarian serous carcinoma: finding from the surveillance epidemiology and end results (SEER) database. J Ovarian Res 2019;12:126.

11. Loizzi V, Rossi C, Cormio G et al. Clinical features of

Cite this article as: Yuan Y, Wang R, Guo F, Zhang Y, Wang $\mathrm{H}$, Li X, Bai GQ. A clinical model to predict the risk of liver metastases in newly diagnosed ovarian cancer: a populationbased study. Transl Cancer Res 2020;9(11):7044-7053. doi: $10.21037 /$ tcr-20-2321 hepatic metastasis in patients with ovarian cancer. Int J Gynecol Cancer 2005;15:26-31.

12. Winter WE 3rd, Maxwell GL, Tian C, et al. Tumor residual after surgical cytoreduction in prediction of clinical outcome in stage IV epithelial ovarian cancer: a Gynecologic Oncology Group Study. J Clin Oncol 2008;26:83-9.

13. Niu GC, Shen CM, Cui W, et al. Hepatic Resection is Safe for Metachronous Hepatic Metastases from Ovarian Cancer. Cancer Biol Med 2012;9:182-7.

14. Luna-Abanto J, Garcia Ruiz L, Laura Martinez J, et al. Liver Resection as Part of Cytoreductive Surgery for Ovarian Cancer. J Gynecol Surg 2020;36:70-5.

15. Clavien PA, Barkun J, de Oliveira ML, et al. The ClavienDindo classification of surgical complications: five-year experience. Ann Surg 2009;250:187-96.

16. Gallotta V, Conte C, D'Indinosante M, et al. Prognostic factors value of germline and somatic brca in patients undergoing surgery for recurrent ovarian cancer with liver metastases. Eur J Surg Oncol 2019;45:2096-102.

17. Gallotta V, Conte C, Giudice MT, et al. Secondary Laparoscopic Cytoreduction in Recurrent Ovarian Cancer: A Large, Single-Institution Experience. J Minim Invasive Gynecol 2018;25:644-50.

18. Zhang C, Guo X, Peltzer K, et al. The prevalence, associated factors for bone metastases development and prognosis in newly diagnosed ovarian cancer: a large population based real-world study. J Cancer 2019;10:3133-9.

19. Barbolina MV. Molecular Mechanisms Regulating OrganSpecific Metastases in Epithelial Ovarian Carcinoma. Cancers (Basel) 2018;10:444.

20. Zecchin C, Gueorguieva I, Enas NH, et al. Models for change in tumour size, appearance of new lesions and survival probability in patients with advanced epithelial ovarian cancer. Br J Clin Pharmacol 2016;82:717-27. 